\title{
A UNIFIED BEHOVIOR OF 2-D AND 3-D NONINVERTIBLE MAP
}

\author{
M. Mammeri \\ Department of Mathematics \\ University of Kasdi Merbah \\ Ouargla, ALGERIA
}

\begin{abstract}
In this paper, the unified behavior for maps with different dimensions is possible from some cases of dynamical systems. This short paper proposes a 2-D noninvertible discrete chaotic map with one bifurcation parameter, and that had only one nonlinear term, and a new 3-D noninvertible discrete chaotic map with twelve bifurcation parameters, and six-nonlinear terms. The two maps they have unified dynamical behavior and can displays same chaotic attractors form same bifurcation parameters and from identical bifurcation route to chaos. This interesting phenomenon is justified by numerical simulation.
\end{abstract}

AMS Subject Classification: $37 \mathrm{C} 29,37 \mathrm{D} 45,37 \mathrm{G} 35$

Key Words: noninvertible map, dynamical behavior, 2-D and 3-D discrete maps, chaotic attractors

\section{Introduction}

Of course, unifying dynamical behaviors for different dimensions of maps are probably rare in dynamical systems, the theoretical research of chaotic behaviors of maps with quadratic inverse and constant Jacobian is a very interesting topic in dynamical systems. One of these models in 2-D is the most famous map, has been intensively studied over past few decades $[1,2,3,4]$. In recent years, there are many documents have studied 3-D chaotic maps such as with quadratic inverse and constant Jacobian $[5,6,7,8,9]$. On the other hand, the

$\begin{array}{lr}\text { Received: } & \text { March 27, } 2017 \\ \text { Revised: } & \text { May 18, } 2017 \\ \text { Published: } & \text { May 23, } 2017\end{array}$

(C) 2017 Academic Publications, Ltd. url: www.acadpubl.eu 
2-D or 3-D noninvertible map is defined as a map with no-constant Jacobian, a few such example are well known, many papers have studied 2-D and 3-D maps with no-constant Jacobian $[10,11,12,13,14,15,16,17,18,19,22]$. So the study of the noninvertible map has important value since, large number of physics, engineering and economics systems have been widely studied found to exhibit a class of noninvertible maps [20, 21, 22]. This short paper investigate a new two simplest 2-D and 3-D noninvertible discrete chaotic maps. The two maps they have an unified dynamical behavior and can exhibits same type of chaotic attractors form identical bifurcation route to chaos. This very interesting phenomenon in dynamical systems is justified by some numerical simulations.

\section{The Proposed 2-D and 3-D Maps}

We consider the two-dimensional polynomial noninvertible map is given as follows:

$$
\left(\begin{array}{c}
\bar{x} \\
\bar{y}
\end{array}\right)=\left(\begin{array}{c}
x-a y \\
x-a x y
\end{array}\right)
$$

Where $(x, y) \in \mathbb{R}^{2}$ and $a \in \mathbb{R}$ is the bifurcation parameter. For $a=0$ the map (1) reduces to two-dimensional linear map, the map (1) is of class $C^{\infty}\left(\mathbb{R}^{2}\right)$, and is one-to-one, the map (1) has only one fixed point is $(0,0)$. Furthermore, this model permits the construction of a new family of attractors dependent on the bifurcation parameter $a$ and initial conditions, as shown in Figures.3. The 2-D map (1) can be extended to a 3-D map.

We consider the new simple three-dimensional polynomial noninvertible quadratic system described by:

$$
\left(\begin{array}{c}
\bar{x} \\
\bar{y} \\
\bar{z}
\end{array}\right)=\left(\begin{array}{c}
x-a y \\
x-b x y \\
f(x, y, z)
\end{array}\right)
$$

Where $f(x, y, z)=a_{0}+a_{1} x+a_{2} y+a_{3} z+a_{4} x^{2}+a_{5} y^{2}-c z^{2}+a_{6} x y+a_{7} x z+a_{8} y z$ and $\left(a, b, c, a_{\mathrm{i}}\right)_{0 \leq i \leq 8} \in \mathbb{R}^{12}$ are bifurcation parameters and $(x, y, z) \in \mathbb{R}^{3}$ are the state variables. Doubtless, map (2) is an extended version of the twodimensional noninvertible discrete chaotic system (1). Furthermore, the map (2) has at most two fixed points are $(0,0, \alpha)$, where $\alpha=\frac{-\left(\mathrm{a}_{3}-1\right) \pm \sqrt{\left(\mathrm{a}_{3}-1\right)^{2}-4 \mathrm{ca}_{0}}}{2 \mathrm{c}}$, if $a_{0}=0$, the origin $(0,0,0)$ is fixed point of the map (2). Figures.4 shows a new family of attractors and chaotic attractors from some cases of the map (2). 


\section{Numerical Investigation}

In this section, we choose eight forms of the map (2) in which the dynamical behaviors of the map (1) and map (2) are identical. Moreover, there are some other cases of the map (2) in which this property holds. The dynamical behaviors of the map (1) and for the map (2) in the cases (2.1) till (2.8) are investigated numerically.

We take the same initial conditions $x_{0}=y_{0}=z_{0}=0.01$, and bifurcation parameters $b=0.8$ and $c=0.1$, let $a$ vary between 0.5 and 1.4. Figures.1(a) and Figures.2(a) shows respectively the identical bifurcation diagrams route to chaos for the state variable $x$ plotted versus increasing the bifurcation parameter $a$ of the map (1) and for the map (2) in the cases (2.1) till (2.8). Also, Figures.1(b) and Figures.2(b) shows respectively the identical diagrams of the largest Lyapunov exponent of the proposed map (1) and for the map (2) in the cases (2.1) till (2.8). Furthermore, Figures.3 and Figures.4 display respectively the same phase portraits of the map (1) and for the map (2) in the cases (2.1) till (2.8).

$$
\begin{gathered}
f(x, y, z)=-c z^{2}, \\
f(x, y, z)=y-c z^{2}, \\
f(x, y, z)=y^{2}-c z^{2}, \\
f(x, y, z)=x+y-c z^{2}, \\
f(x, y, z)=x+y^{2}-c z^{2}, \\
f(x, y, z)=x+y+y^{2}-c z^{2}, \\
f(x, y, z)=x+y+x^{2}+y^{2}-c z^{2}, \\
f(x, y, z)=1+x+y+x y+y^{2}-c z^{2} .
\end{gathered}
$$




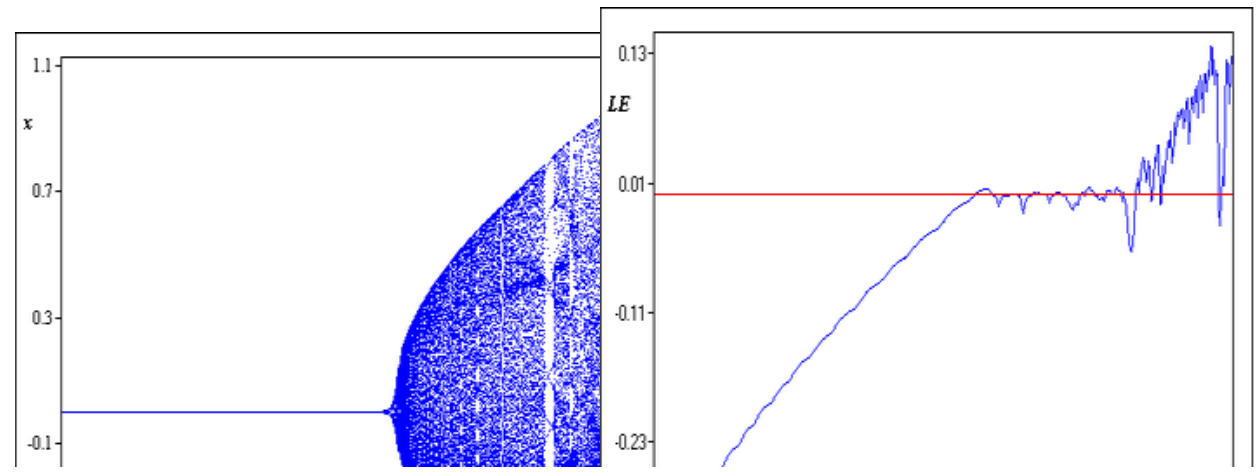

Figure 1(a): Bifurcation diagram Figure 1(b): Lyapunov expoof the map (1) of state $x$ versus nents of the map (1) versus $0.5 \leq$ $0.5 \leq a \leq 1.4$ $a \leq 1.4$.

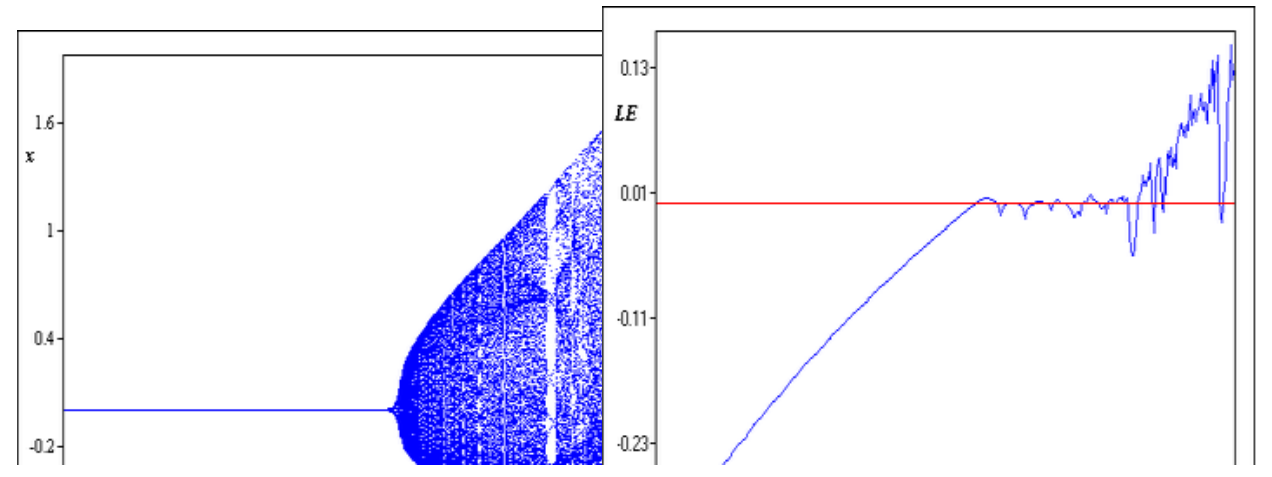

Figure 2(a): Bifurcation diagram Figure 2(b): Lyapunov expoof the map (2) of state $x$ versus nents of the map (2) versus $0.5 \leq$ $0.5 \leq a \leq 1.4$ and $b=0.8, c=a \leq 1.4$ and $b=0.8, c=0.1$. 0.1 . 


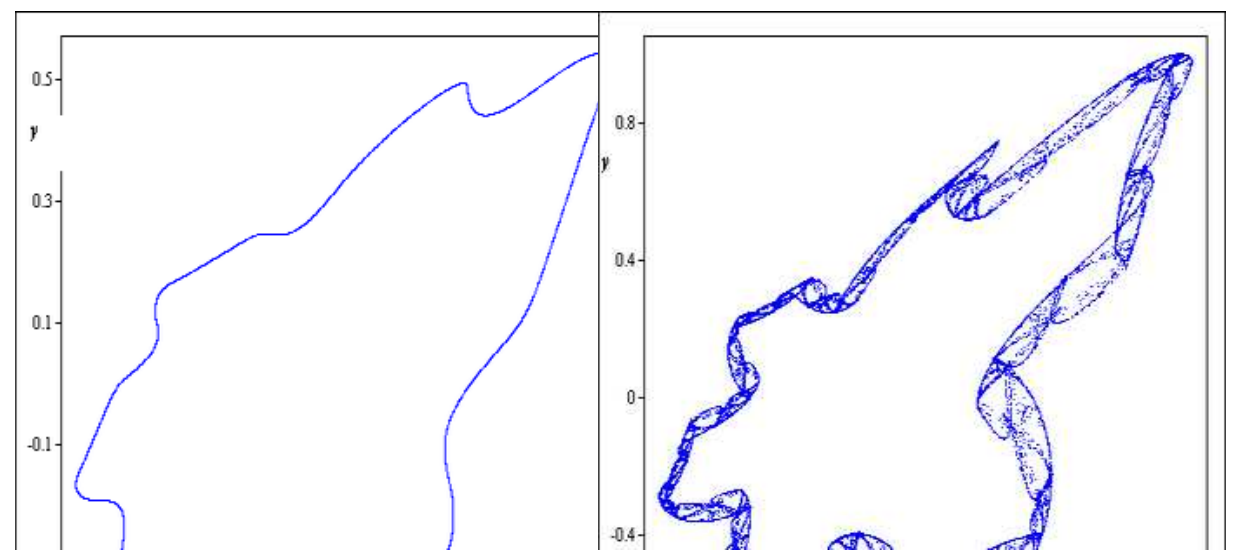

Figure 3(a): Periodic attractor of Figure 3(b): Chaotic attractor of the map (1) $(a=1.218)$. the map (1) $(a=1.26)$.

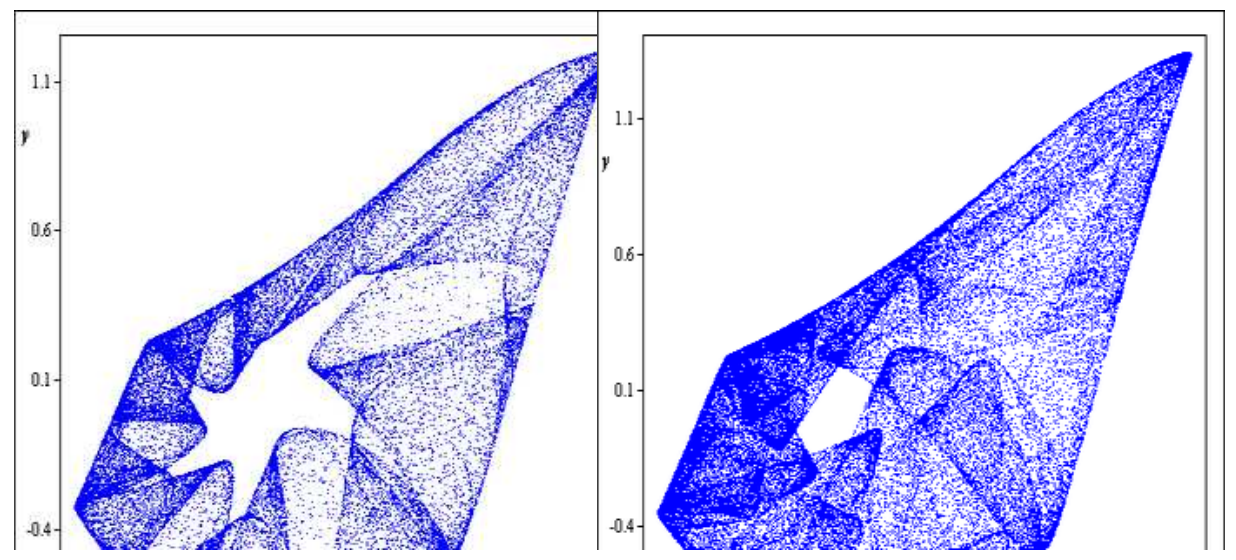

Figure 3(c): Chaotic attractor of the map (1) $(a=1.302)$.

Figure 3(d): Chaotic attractor of the map (1) $(a=1.33)$. 


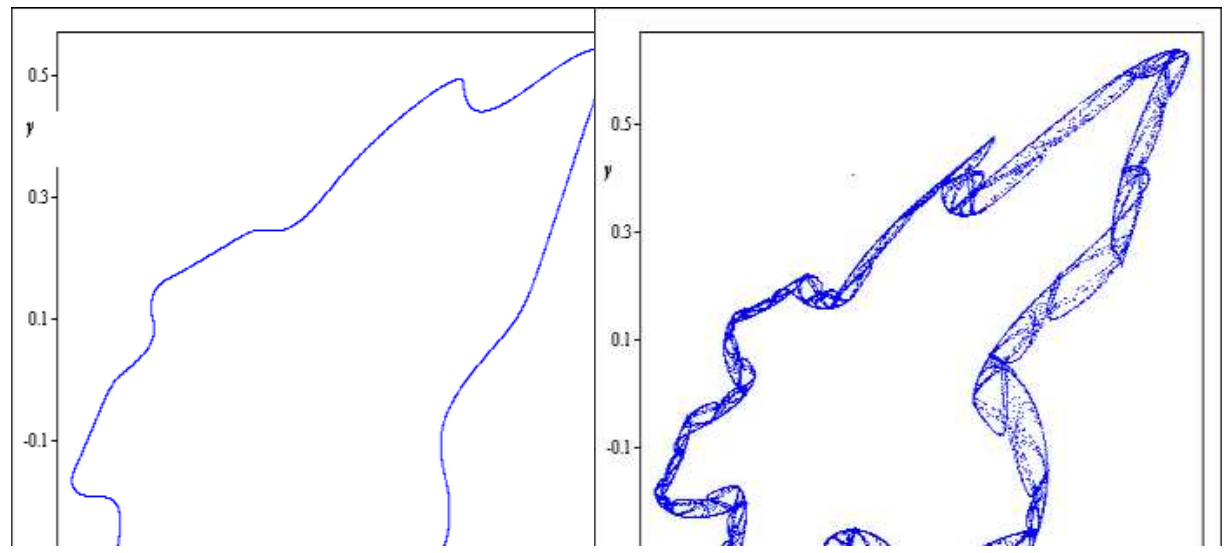

Figure 4(a): Periodic attractor of Figure 4(b): Chaotic attractor of the map $(2)(a=1.218, b=0.8$ the map $(2)(a=1.26, b=0.8$ and $c=0.1)$. and $c=0.1)$.

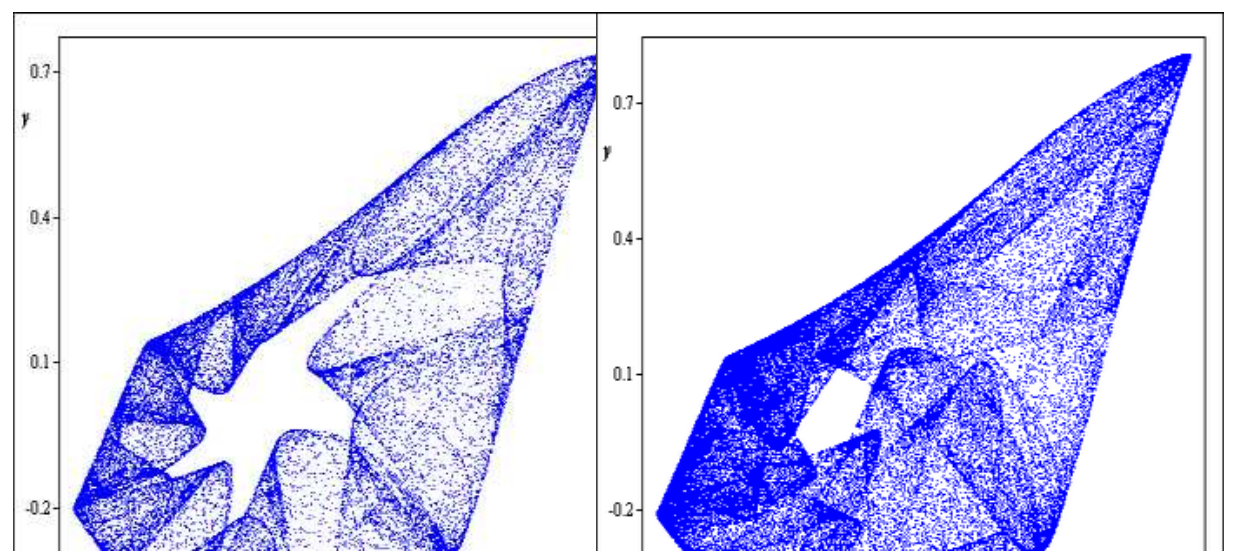

Figure 4(c): Chaotic attractor of the $\operatorname{map}(2)(a=1.302, b=0.8$ and $c=0.1)$.
Figure 4(d): Chaotic attractor of the map $(2)(a=1.33, b=0.8$ and $c=0.1)$.

\section{Conclusion}

This short paper investigate a new two simplest 2-D and 3-D noninvertible discrete maps that realizes a new phenomenon in which the dynamical behaviors for our proposed map (1) and map (2) in the cases (2.1) till (2.8) are unified and 
they have same chaotic attractors form same bifurcation parameters and from identical bifurcation route to chaos, this important phenomenon is obtained by numerical investigation.

\section{References}

[1] M. Hénon, A two-dimensional mapping with a strange attractor, Comm. Math. Phys.50 (1976), no. 1, 69-77.

[2] M. Benedicks and L. Carleson, The dynamics of the Hénon map, Ann. of Math. (2)133(1991), no. 1, 73-169.

[3] F. R. Marotto, Chaotic behavior in the Hénon mapping, Comm. Math. Phys. 68 (1979), no. $2,187-194$.

[4] M. A. Aziz-Alaoui, C. Robert, and C. Grebogi, Dynamics of a Hénon-Lozi-type map,. Chaos Solitons Fractals12 (2001), no. 12, 2323-2341.

[5] S. V. Gonchenko, I. I. Ovsyannikov, C. Simó, D. Turaev., Three-Dimensional Hénon-like Maps and Wild Lorenz-like Attractors., International Journal of Bifurcation and Chaos., 15 (11), (2005), 3493-3508.

[6] S. V. Gonchenko, J. D. Meiss, I. I. Ovsyannikov., Chaotic Dynamics of Three-Dimensional Hénon Maps that Originate from a Homoclinic Bifurcation., Regular and Chaotic Dynamics., 11 (2), (2006), 191-212.

[7] S. V. Gonchenko, V. S., Gonchenko., and J. C. Tatjar., Bifurcation of Three-Dimensional Diffeomorphisms Non-Simple Quadratic Homoclinic Tangencies and Generalized Hénon Maps., Regul. Chaotic Dyn., 12 (3), (2007), 233-266.

[8] L. P. Shilnikov, D.V.Turaev., An example of a wild strange attractor., Mat. Sb., 189 (1998), 137-160. Engl. transl: Sb. Math., 189, (1998), 291-314.

[9] Gonchenko S.V., Shilnikov L.P. and Turaev D.V., On global bifurcations in threedimensional diffeomorphisms leading to wild Lorenz-like attractors., Regul Chaotic Dyn., 14 (1), (2009), 137-147.

[10] Zeraoulia Elhadj, J. C. Sprott., A minimal 2-D quadratic map with quasi-periodic route to chaos, International Journal of Bifurcation and Chaos, Vol. 18, No. 5 (2008) 1567-1577.

[11] R. Lozi., Un attracteur étrange du type attracteur de Hénon, Journal de Physique, Colloque C5, supplément, 8, (1978), 39, 9-10.

[12] L. J. Wallen., One parameter groups and the characterization of the sine function., Proc. Amer. Math. Soc., 102, (1988), 59-60.

[13] J.-H. Kim, Y.-S. Chung, S.-Y. Chung., Generalization of characterizations of the trigonometric functions., Math. Proc. Camb. Phil. Soc., 141, (2006), 409-519.

[14] S. Banerjee, S. Parui, Delays in PWM control loops imply discontinuity in sampled data models of power electronic circuits., in Proc. International Symposium on Circuits and Systems., vol. 3, (2003), III-92-III-95.

[15] Bi Chuang, Q. Zhang, and al., Bifurcation and attractor of two-dimensional sinusoidal discrete map, Acta Phys Sin., 62 (24), (2013), 240503-1-240503-7. 
[16] E. Zeraoulia, J. C. Sprott., On some universal dynamics of a 2-D Hénon-like mapping with an unknown bounded function., Annual Review of Chaos Theory, Bifurcations and Dynamical Systems., Vol. 1, (2012), 50-60.

[17] W. Hu, G.-H. Zhao, G. Zhang, and al., Stabilities and bifurcations of sine dynamic equations on time scale., Acta Phys. Sin., vol. 61 (17), (2012), 170505-1-170505-12.

[18] K. Mandal, S. Banerjee, C. Chakraborty., "Symmetry-Breaking Bifurcation in SeriesParallel Load Resonant DC-DC Converters.," IEEE Trans. Circuits Syst. I., vol.60, no.3, (2013), 778-787.

[19] K. Manda, S. Banerjee, C. Chakraborty., Symmetry-breaking bifurcation in load resonant dc-dc converters., in Proc. International Symposium on Circuits and Systems., (2011),1327-1330.

[20] S. Banerjee, G. C. Verghese., Nonlinear phenomena in power electronic: Attractor, Bifurcation, Chaos, and Nonlinear Control, IEEE Trans, New York, 2001.

[21] T. K. Tse,. Complex behavior of switching power converters, CRC Press, Boca Raton, USA, (2003).

[22] G. I Bschi, F. Tramontana., Three-dimensional discrete-time Lotka-Volttera models with an application to industrial clusters,. Commun N-Sci Numer Simulat 15, (2010), 30003014 . 